\title{
Editorial
}

\section{Functional Carbon Nanomaterials}

\author{
Myoung-Woon Moon, ${ }^{1}$ Ho-Young Kim, ${ }^{2}$ Aiying Wang, ${ }^{3}$ and Ashkan Vaziri ${ }^{4}$ \\ ${ }^{1}$ Korea Institute of Science and Technology (KIST), Seoul 136-791, Republic of Korea \\ ${ }^{2}$ Seoul National University, Seoul 151-744, Republic of Korea \\ ${ }^{3}$ Ningbo Institute of Materials Technology and Engineering (NIMTE), Chinese Academy of Sciences, Ningbo 315201, China \\ ${ }^{4}$ Northeastern University, Boston, MA 02155, USA
}

Correspondence should be addressed to Myoung-Woon Moon; mwmoon@kist.re.kr

Received 4 January 2014; Accepted 4 January 2014; Published 10 February 2014

Copyright (c) 2014 Myoung-Woon Moon et al. This is an open access article distributed under the Creative Commons Attribution License, which permits unrestricted use, distribution, and reproduction in any medium, provided the original work is properly cited.

Carbon nanomaterials have increasingly gained interest due to their capability of forming various allotropes including nanotubes, fullerenes, diamond, amorphous carbon, and more recently, graphene. Carbon nanomaterials or nanostructures offer exceptional flexibility in tailoring various properties for specific purposes due to their chemical inertness. For example, they have resistance to acidic or basic media, structural stability at high temperatures in the absence of air, and tunable chemical nature of hydrophobicity. Different physical forms of carbon materials in nanoscale, including thin films, graphene foams or sponges, carbon nanotube forests, carbon fibers, carbon nanowalls, and porous carbon materials, can lead to a variety of functions. In addition to chemical and physical modifications, the functions of carbon nanomaterials can be altered by adding or doping metal elements such as gold, platinum, or silver. Carbon nanomaterials have been widely applied for use in energy, environment, water, biomedicine, and other fields.

The major goals of this special issue are to find novel fabrication methods for functional carbon nanomaterials and the modification or nanostructuring of carbon surfaces for novel functionalization in up-to-date applications. This issue includes research papers covering a wide range of current progress on the modification with physical and chemical methods and characterization for functional carbon nanomaterials and nanostructures, as well as the related applications in hard coatings, energy storage and conversion, water filtration, catalysts, and decontamination. The combined study on novel experimental preparation of carbon nanomaterials such as carbon nanotubes, amorphous carbon films, and fullerenes and the theoretical investigations of functional carbon nanomaterials with first-principles/ab initio calculation are presented. Moreover, in this special issue, some papers are particularly invited to address the above mentioned issues.

One paper of this special issue presents the functional coating of $\mathrm{W}$-incorporated diamond-like carbon $(\mathrm{W}-\mathrm{C}: \mathrm{H}$ ) films fabricated by a hybrid beams system consisting of a DC magnetron sputtering and a linear ion source. It shows that the films mainly exhibited the feature of amorphous carbon when $\mathrm{W}$ concentration in the films was less than 4.38 at.\%, where the incorporated $\mathrm{W}$ atoms would be bonded with $\mathrm{C}$ atoms and resulted in the formation of nanoparticles along with a minimum value of residual compressive stress, a higher hardness, and better tribological properties.

Most papers in this special issue provide the functionalization of carbon nanotubes with doping and special modifications. Another paper explores field emission properties of carbon nanotubes (CNTs) with boron doping and $\mathrm{H}_{2} \mathrm{O}$ adsorption using ab initio calculations. They investigated the electron field emission performance of CNTs simultaneously adsorbed with one $\mathrm{H}_{2} \mathrm{O}$ molecule and doped with one boron atom $\left(\mathrm{BCNT}+\mathrm{H}_{2} \mathrm{O}\right)$. The results indicate that the electrons are localized at the top of $\mathrm{BCNT}+\mathrm{H}_{2} \mathrm{O}$ and the electronic density of states (DOS) around the Fermi level is enhanced. A different paper addresses nitrogendoped CNTs synthesized by pyrolysis of (4-\{[(pyridine-4yl)methylidene]amino\}phenyl) ferrocene in a solution of either acetonitrile or toluene as carbon source. Acetonitrile was found to produce mainly N-CNTs with "bamboo" morphology while toluene formed a mixture of pristine CNTs 
and N-CNTs in the ratio of $1: 1$. The authors of another paper addressed adsorption of Eu(III) on multiwalled CNTs in terms of effects of $\mathrm{pH}$, ionic strength, solid-liquid ratio, and water-soluble fullerene. The sorption process was influenced strongly by $\mathrm{pH}$ changes and ionic strength. An additional paper of this issue reports a comparative investigation of the antibacterial activity of two water-soluble fullerene derivatives bearing protonated amine $(\mathrm{AF})$ and deprotonated carboxylic groups appended to the fullerene cage via organic linkers. It reveals that the water-soluble cationic fullerene derivative AF possesses promising antibacterial activity, which might be utilized in the development of novel types of chemical disinfectants.

One more paper addresses decontamination of surfaces exposed to carbon-based nanotubes and nanomaterials. Contamination of surfaces by nanomaterials can happen due to accidental spillage and release or gradual accumulation during processing or handling. The article proposes and investigates a potential method for surface decontamination of carbon-based nanomaterials using solvent cleaning and wipes. The results show that the removal efficiencies for single- and multiwalled carbon nanotubes from silicon wafers sprayed with water-surfactant solutions prior to mechanical wiping are greater than $90 \%$ and $95 \%$, respectively. The need for further studies to understand the mechanisms of nanomaterial removal from surfaces and development of standard techniques for surface decontamination of nanomaterials is highlighted.

\title{
Acknowledgments
}

The editors would like to thank the authors for their contributions to this special issue and the reviewers for their time and dedication.

\author{
Myoung-Woon Moon \\ Ho-Young Kim \\ Aiying Wang \\ Ashkan Vaziri
}



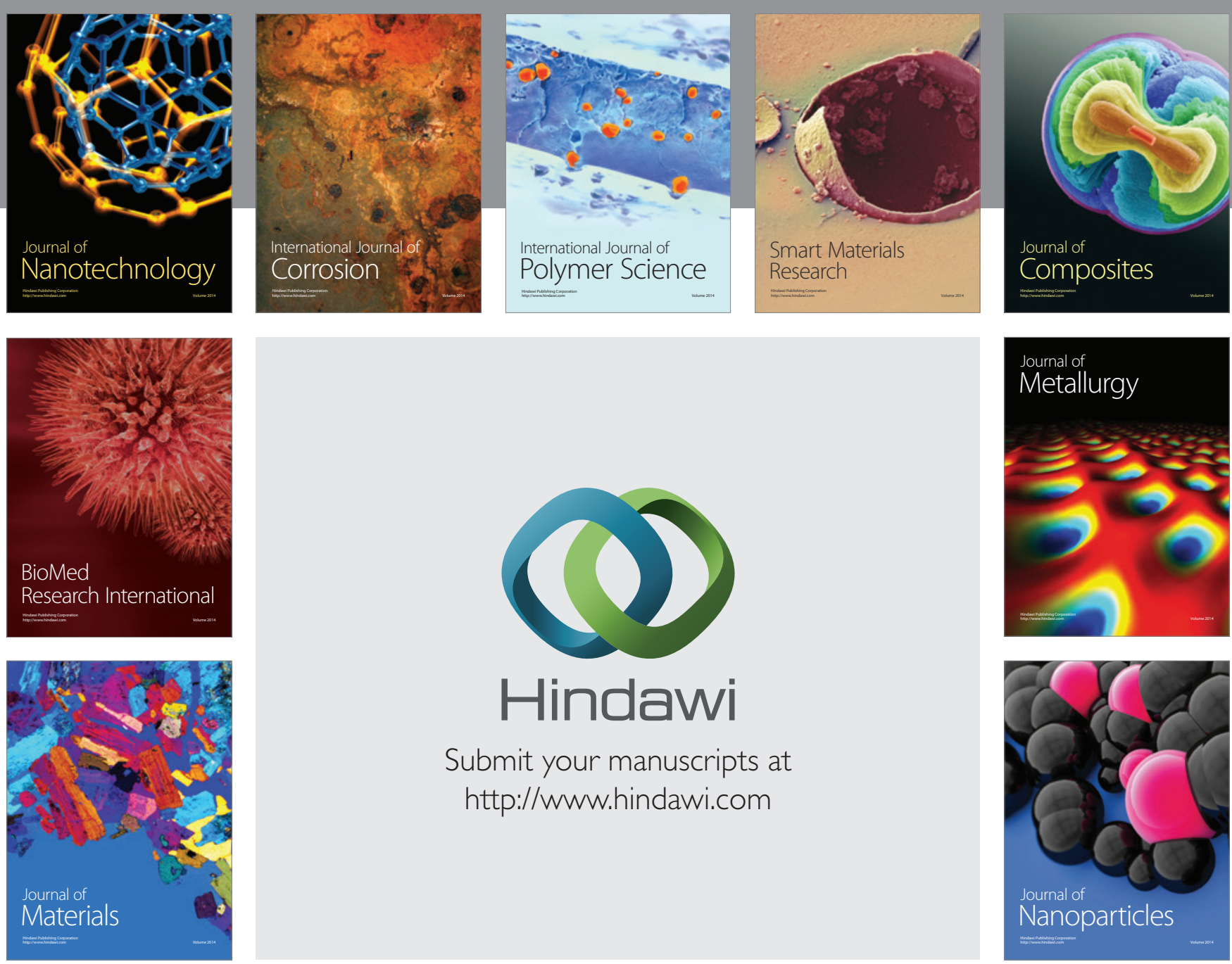

Submit your manuscripts at http://www.hindawi.com
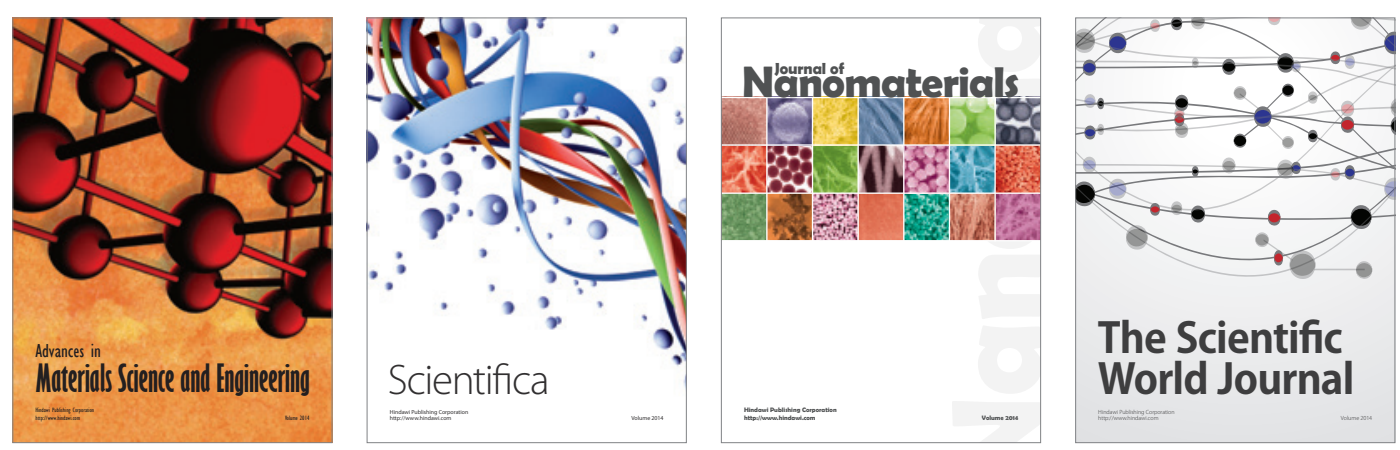

\section{The Scientific World Journal}
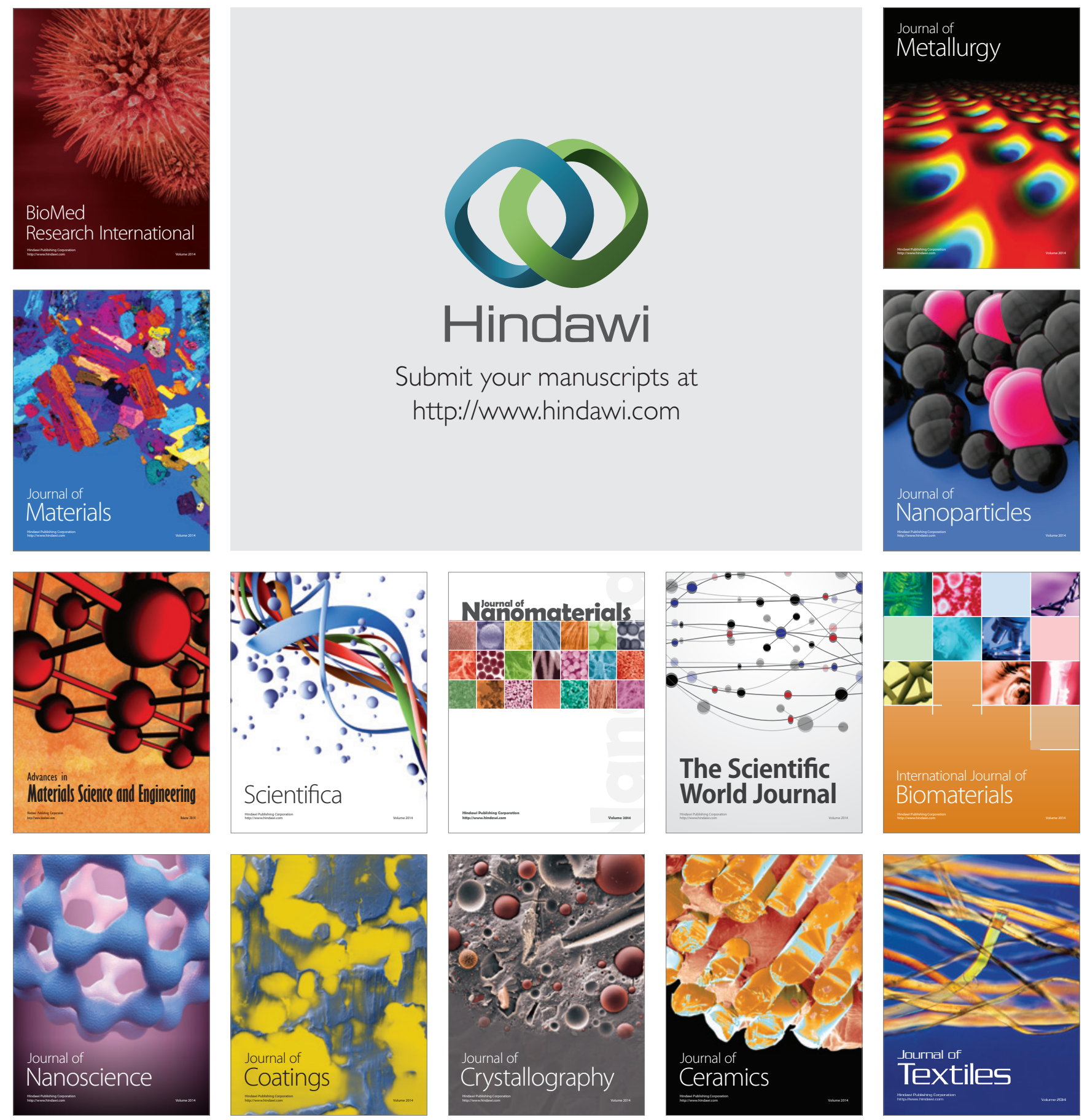